\title{
Der Schaffhauser Stadtarzt Johann Cosmas Holzach (1518-1595) und seine Schrift «Prob des Uszsatzes»
}

\author{
Von Marie-Louise Portmann
}

Johann Cosmas Holzach, dessen Schrift über den Aussatz im Mitte]punkt unserer kleinen Betrachtung steht, entstammte einem angesehenen Basler Geschlecht, das schon im 13. Jahrhundert in Basel ansässig war. Der Großvater, Eucharius Holzach, der 1521 starb, war Schultheiß im Klein-Basel und Herr zu Großhüningen, das er von Österreich zu Lehen trug und 1521 an Basel abtrat ${ }^{1}$. Der Vater, der ebenfalls den Namen Eucharius trug und von 1486 bis 1558 lebte, war trotz seines etwas lockeren Lebenswandels in Basel ein angesehener Arzt, der wegen seines prunkvollen Auftretens in dem jungen Felix Platter den Wunsch weckte, ebenfalls Medizin zu studieren und es auch soweit zu bringen. Platter schreibt in seiner Autobiographie: «So bewegt mich auch nit wenig dohin, daß ich D.Sebastianum Sinkeler, D.Eucharium Holtzach gesach in schamleten röcken, mit sammat breit umleit, herumbziechen und bey den leuten groß ansechen haben ... ${ }^{2}$ Wie aus der von Alfred Hartmann herausgegebenen Amerbach-Korrespondenz zu ersehen ist, stand Holzach in enger Beziehung zur Familie Amerbach ${ }^{3}$; war er doch in Schlettstadt auf dem Gymnasium zusammen mit den älteren Amerbach-Söhnen Bruno und Basilius, dann mit ihnen zusammen in Paris und ging dann nach Montpellier, wo er Mitte 1511 doktorierte. Nach seiner Promotion ließ er sich in Basel als praktischer Arzt nieder und bewohnte den geräumigen Ziegelhof, den heutigen Hattstädter Hof in Klein-Basel.

Hier wurde um 1518 Johann Cosmas, der spätere Schaffhauser Stadtarzt geboren. Er hatte zahlreiche Geschwister, denn Eucharius Holzach hatte 13 Kinder aus zwei Ehen. Johann Cosmas war der zweite Sohn aus der Ehe mit Veronika Rispach. Seine ersten Schuljahre verbrachte er wohl in Basel, doch mit 15 Jahren kam er zum Studium nach Paris, wie aus der Amerbach-Korrespondenz zu entnehmen ist ${ }^{4}$. Durch Vermittlung von

1 Historisch-Biographisches Lexikon der Schweiz, Band 4, S.279, Neuenburg 1927.

${ }^{2}$ Felix Platter, Autobiographie, herausgegeben von D.A. Fechter, S. 129, Basel 1840.

3 Die Amerbachkorrespondenz, herausgegeben von Alfred Hartmann, Bände I-IV, Basel 1942 ff. Briefe Nr. 65, 127, 130, 154, 160, 186, 201, 246, 265, 270, 277, 281, 314, 447.

4 Ebenda, Briefe Nr. 1774, 1780, 1951. 
Bonifatius Amerbach wurde er bei Johannes Sturm untergebracht, dem nachmals berühmten Organistor des Schulwesens in Straßburg und bedeutenden Vertreter des pädagogischen Humanismus. Wann und wo Holzach zum Doktor der Medizin promoviert wurde, ist nicht bekannt, doch muß dies vor 1549 geschehen sein. Denn in diesem Jahr, am 23. Juli 1549, heiratete Dr.med.Johann Cosmas Holzach in Schaffhausen Gertrud von Waldkirch, die Tochter des Bürgermeisters Junker Johannes von Waldkirch und dessen Ehefrau Gertrud Grebel von Baden ${ }^{5}$. Holzach scheint sich schon vor oder nach seiner Heirat in Schaffhausen niedergelassen zu haben. Sämtliche Kinder, drei Söhne und fünf Töchter, sind jedenfalls in Schaffhasuen geboren worden ${ }^{6}$. Am 25. Februar 1559 wurde Holzach auch Bürger von Schaffhausen ${ }^{7}$, und seit Pfingsten 1559 ist er in den jährlichen Ratsrödeln der Stadt als Arzt eingetragen ${ }^{8}$. Seit 1570 war er überdies Schulherr ${ }^{9}$, seit 1581 Eherichter und seit 1592 Mitglied des Ausschusses «die armen krankhen presthaften lüth zu beschowen ». ${ }^{10} \mathrm{Zu}$ diesem Amt war er besonders qualifiziert, hatte er doch schon 1558 seine Schrift Prob des Uszsatzes verfaßt, auf die ich nachstehend zu sprechen komme.

Über Holzachs ärztliches Wirken erfahren wir manches aus den 15 Briefen, die Conrad Geßner, der berühmte Zürcher Gelehrte, vom 22.Juni 1553 bis zum 24. September 1564 an den Schaffhauser Arzt schrieb, und die Kaspar Wolff nach Geßners Tod 1577 bei Froschauer in Zürich zusammen mit vielen anderen Briefen Geßners herausgab ${ }^{11}$. Geßner und Holzach tauschten Erfahrungen und Rezepte aus, und aus den Briefen geht hervor, daß der Zürcher Arzt die Ratschläge seines Schaffhauser Freundes stets sehr hoch einschätzte. Den Hauptbestandteil der medizinischen Verordnungen sowohl Geßners wie auch Holzachs bildeten die Kräuter, und jeder besaß einen Garten, in dem er die verschiedensten Pflanzengattungen zum Gedeihen zu bringen suchte. Die beiden Freunde tauschten denn auch

5 Staatsarchiv Schaffhausen, Ehebuch 10.

6 Zivilstandsamt Schaffhausen, Taufbuch 1540-1592. Eucharius, getauft am 9. Juli 1550, Gertrud am 5. Februar 1552, Anna am 12. Oktober 1553, Veronika am 18. Februar 1555, Damian am 23. August 1556, Justina am 25. Juli 1558, Eucharius am 11. August 1560 und Elsbeth am 7.Dezember 1573.

7 Staatsarchiv Schaffhausen, Bürgerrecht A 1, 31 v.

8 Staatsarchiv Schaffhausen, Ratsprotokolle 19, XXVII r.

9 Ratsprotokolle 30, XIII r. 31, 9 r. Schulratsprotokolle 1, 30 r.

10 Ratsprotokolle 31, 9 v, 52, XIII v, 55, VI v, VII r. XI v, XVI v.

11 Epistolarum medicinalium Conradi Gesneri medici et philosophie Tigurini libri III, Liber III, S. 81-88, Zürich 1577. 
häufig Sämereien aus, und da konnte denn Holzach, der sich auch aus Schaffhausens berühmtem Kräutergarten bedienen konnte, seinem Zürcher Kollegen zu mancher seltenen Pflanze verhelfen. Wie sehr Holzachs Therapie auf pflanzlichen Heilmitteln beruhte, ist aus einem handschriftlich erhaltenen Brief an die Paradieser Klosterfrau Afra Blank ersichtlich, der vom 21. September 1575 datiert ist und der im Staatsarchiv Schaffhausen aufbewahrt wird. Der Patientin, die offenbar an Koliken litt, riet Holzach: «... sollend süden lassen in wasser und wißem win Camillen blümlin, fenchelkrut, Steinkleen, Käspappel, und flachs samen zerknütscht. Diß gesotten wasser aldan sihen durch ein tüchlin, und woll ußtrucken, und in ein suw blatteren thun, und in zimlicher werme überschlahen, wo sich der schmertzen und grimmen im lyb thut erregen.» Weitere handschriftliche Briefe Holzachs an Bonifatius Amerbach, Felix Platter und Ulrich Iselin werden in der Basler Universitätsbibliothek aufbewahrt. Holzach starb in Schaffhausen am 15. Juni 1595 und wurde im Stiftsgarten des dortigen Münsters beigesetzt. Sein mit dem Familienwappen der Basler Holzach geschmücktes Epitaph und dasjenige seiner Tochter Anna von 1629 hängt jetzt im Kreuzgang des Münsters zu Schaffhausen.

Den Angaben älterer Lexika (z.B. von Christian Gottlieb Jöcher, Hans Jakob Leu) ist zu entnehmen, daß Holzach mehrere Werke verfaßt haben soll. Jedoch konnte ich nur seine 1558 bei Froschauer in Zürich erschienene Schrift Prob des Uszsatzes ermitteln. Eine lateinische Ausgabe Experimentum et examen leprae sowie die angeblich in Lyon erschienenen Annotationes in Dioscoridem waren trotz intensiver Nachforschungen nirgends aufzufinden, und ich bin mit dem Schaffhauser Staatsarchivar Herrn Dr. Hans Lieb der Meinung, daß es sich hier vermutlich um «bibliographical ghosts» handelt.

Doch wenden wir uns nun der Schrift Holzachs über den Aussatz zu. Es handelt sich dabei um eine klar gegliederte Abhandlung über die Symptome, an denen der Aussatz zu erkennen sei. Sie ist in deutscher Sprache verfaßt, da sie für die nicht akademisch gebildeten Scherer und Chirurgen bestimmt war, denen unter anderem auch die Aufgabe oblag, durch eine Beschauung festzustellen, ob ein Patient an Aussatz erkrankt und deshalb abzusondern sei. Eine solche Aussatz-Schau hat der deutsche Chirurg Hans Gersdorf, der in seinem Feldtbuch der Wund-Artzney, Straßburg 1517, erstmals in deutscher Sprache über den Aussatz schrieb, im Bilde darstellen lassen. Holzach prägt nun seinen Lesern ein, ihr Urteil nicht nur auf ein einziges Symptom hin zu fällen, sondern sich immer vor Augen zu halten, 
ob die meisten der genannten Anzeichen der «Malazey», wie er die Lepra auch nennt, vorhanden seien oder gar alle zusammen. Als Humoralpathologe erblickt Holzach die Ursache der Lepra wie Oreibasios von Pergamon ${ }^{12}$, der um 360 n. Chr. lebte, und Paulus von Aegina ${ }^{13}$, der im 7.Jahrhundert wirkte, in einem Überschuß an schwarzer Galle. Man solle sich deshalb erkundigen, was der Kranke zu essen pflegte, «ob villicht söliche spyß und tranck ein grobe/trübe/melancolische füchtigkeit im lyb volbringen unn gebären möchtind ...» Ganz in der mittelalterlichen Tradition steht Holzach, wenn er, wie er beiläufig bemerkt, vier Arten der Lepra unterscheidet. Diese Einteilung in vier Arten geht auf Abulcasis zurück, der um 1000 n. Chr. in Cordoba lebte ${ }^{14}$. Mit wenigen Varianten übernahmen dann auch die salernitanischen Ärzte dieses Schema von Abulcasis, wobei die schwarze Galle, wie man glaubte, je nachdem entweder allein, oder mit einem Übermaß an Schleim, Blut oder gelber Galle eine der vier Arten des Aussatzes erzeugte ${ }^{15}$. Doch die Unterscheidung der vier Arten durch die Chirurgen hält Holzach nicht für wichtig, wie er denn auch keine Therapie angibt, was allein Sache der Ärzte war. Eine wichtige Aufgabe der Scherer war die Blutprobe. Es wurde ein Aderlaß vorgenommen und das Blut untersucht. Ist der Patient aussätzig «wirt (das blut) erfunden stincket, schwartz, grob, zäch / unn geriben mit den fingern / ruch / als ob es voll sand oder grieß. Welches auch heiter und klarlich ersehen wirt / wenn es ... mit reinem luterem wasser durch ein dick linin tuch gesigen wirt. So auch saltz under sölichs blut geworffen / zerschmiltzt nit lychtlich. Deßglychen essig uff sölich gstanden blut gossen / wallt uf / glych als beschicht/ wenn er uff den herd geschütt: welche prob in diesem handel nit zeunderlassen.» Eine ganz ähnliche Blutprobe hatte schon Gersdorf in seiner Wundarznei beschrieben, doch Holzach warnt davor, allein auf dieses Zeichen zu achten, da es auch bei andern Krankheiten auftreten könne. Erst Felix Platter erkannte dann, daß alle diese Blutproben wertlos seien ${ }^{16}$.

12 Oreibasios, Collectio medica, Buch XLV, Kap. 27. herausgegeben von Ch. Daremberg, Paris 1851, Band IV, S. 59 ff.

13 Paulus von Aegina, Buch IV, Kap. 2, in Corpus Medicorum Graecorum, Band IX/1, herausgegeben von I.L. HeIBERG, Leipzig/Berlin 1921, S. 321.

14 Abulcasis, Liber theoricae nec non practicae, tract. 31, Kap. 2, Augustae Vindelicorum 1519.

15 Antoinette Stettler-Schär, (unveröffentlichtes Manuskript), Die Leprologie des Mittelalters.

16 Felix Platter, Prax. med., Buch III, De Defoedatione, Kap. 4, ed. Felix Platter nepos, Basileae 1656. 


\section{Slůt/barti/tnoll/otůpett/glyber fül/}

Des atbems gftanck/ono zeycl)en vill/

fiirwar reo ib/oie zótgen an/

Das difer fey cin malbig man.

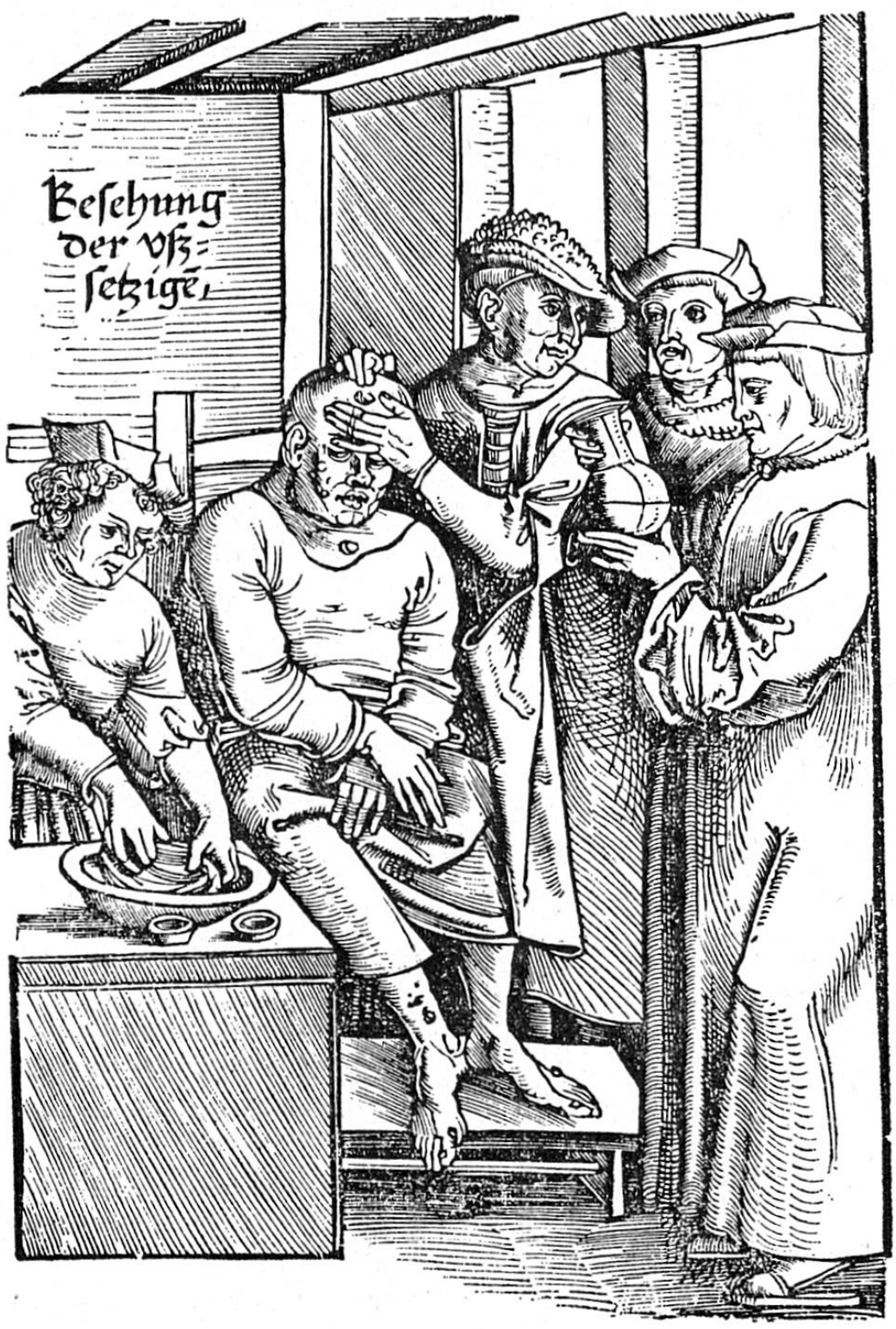

Aus: Hans Gersdorf, Feldtbuch der Wund-Artzney, Seite 168, Straßburg 1540 
Wie Sie wissen, unterscheidet man heute zwei Haupttypen der Lepra, nämlich den Knollenaussatz (lepromatöse Lepra) und den Nervenaussatz (tuberkuloide Lepra). Eines der wichtigsten Symptome des Nervenaussatzes sind die Sensibilitätsstörungen, die schon von den salernitanischen Ärzten diagnostiziert wurden. Holzach legt denn auch auf die folgende Probe größten Wert: «Nach der lässin (dem Aderlaß) / sol die person / so die ougen wol verbunden / an versenen/oder anderen orten des lybs / ungewarnter sach gestupfft werden / unn sy fragen / ob sy etwas empfunden. Dann wo nit / ists mißlich.» Doch auch Unempfindlichkeit sei kein sicheres Anzeichen für Aussatz, meint Holzach, da sie auch bei andern Krankheiten vorkommen könne.

Wie schon Guy de Chauliac unterscheidet auch Holzach mehrdeutige und eindeutige Symptome ${ }^{17}$. Auf die eindeutigen Symptome geht der Schaffhauser Arzt im zweiten speziellen Teil seiner Schrift ein. Am augenfälligsten zeigt sich der Aussatz, nach Holzach, in einer Veränderung des Angesichts. Dann beschreibt er in seiner kernigen Sprache das so charakteristische Bild der Facies leonina, um einen modernen Ausdruck zu gebrauchen. Sie kennzeichnet den Knollenaussatz. Knotenförmige Infiltrate der Stirn- und Augenbrauengegend mit meist frühzeitigem Ausfall der Augenbrauen, hochgradige Verdickung der Wangen, Lippen, Nase und Ohrläppchen fallen in die Augen. Holzach gibt den Chirurgen die Anweisung, beim Angesicht darauf zu achten, «ob es schützlich / mit einer schwartzen röte / mit vil knorren unnd blätterlin: und die stirnen erschyne / ob sy gespannen sey.» An den Augen erkennt schon Holzach die Bindehautentzündung als Begleiterscheinung des Aussatzes. Auch soll der Beschauer darauf achten, «ob die augbrauwen ufgeblasen / unn ire härlich ußfallind / und an statt kleine und gar nach unschynbare wachsind.» Sehr wichtig ist das Aussehen der Nase. Es soll festgestellt werden, «ob sy ußwendig in dicke wachse / unn inwenig ynfalle. Ob die krösplen (der Knorpel) darinnen zernagt / unnd inwendig ein verseerung erschyne: welches / so die naßlöcher ufgespert / ersehen magst. » Auch das Übergreifen der Krankheit auf die Schleimhaut der oberen Luftwege erzeugt Symptome. So sieht Holzach in der heiseren Sprache, verbunden mit Atembeschwerden ein Anzeichen für Lepra, sofern die andern Symptome auch vorhanden sind. Die Muskelatrophie, ein Befund bei Nervenausatz, wird ebenfalls von Holzach genannt. So soll der Untersuchende sehen, «ob die Müß / in Latin

17 Guy de Chauliac, Chirurgia magna, Tract. VI, Doctr. I, Kap. 2, Lyon 1585, S. 251-258. 
Musculi genannt / verzert / und yngefallen / sonderlich an waden / und zwüschen dem dumen unnd zeiger.» Die Muskelatrophie zwischen Daumen und Zeigefinger bei Aussatz wurde erstmals von Bernard de Gordon beobachtet, der seit etwa 1280 in Montpellier lehrte ${ }^{18}$. Doch betont Holzach auch hier, daß dieses Zeichen für sich allein genommen nicht unbedingt auf Aussatz hindeute. Ein wichtiges, jedoch mehrdeutiges Zeichen ist sodann ein Exanthem am ganzen Körper. «... So die person ein jucken und byssen am gantzen lyb empfunde / so die hut vast gespannen, desglychen mit zittermälen / schüppen / mit einer kretzigen / stübenden rud / oder anderen macklen und fläcken verunreiniget / » und «vil knöpff oder knorren / unnd sonderlich am angesicht / erfunden: welches für ein gar mißlich zeichen zehalten.»

Wenn wir uns nun zum Schluß die Frage stellen, ob auf Grund von Holzachs Angaben die sichere Diagnose einer Lepra möglich war, so müssen wir sagen, daß in Anbetracht des Fehlens jeglicher bakteriellen Untersuchungsmethoden sicher viele Fehldiagnosen gestellt wurden, doch nehmen wir alle Symptome zusammen, die Holzach im speziellen Teil seiner Schrift aufzählt, so hatte doch eine Diagnose nach Holzachs Angaben eine hohe Wahrscheinlichkeit. Wenn der Schaffhauser Arzt auch kein Pionier der Lepraforschung war, so gebührt ihm doch das Verdienst, die zu seiner Zeit gültigen Anzeichen des Aussatzes in klarer und verständlicher Darstellung der Nachwelt überliefert zu haben.

Für mancherlei Auskünfte bin ich dem Staatsarchivar von Schaffhausen, Herrn Dr. HANS LIEB, sehr zu Dank verpflichtet.

18 Bernard de Gordon, Opus Lilium medicinae, Lyon 1574 (vgl. Manuskript von ANtoiNETTE STETTLER-SCHÄR). 